Technological University Dublin

ARROW@TU Dublin

2010-01-01

\title{
Design and Fabrication of Holographic Optical Elements for Applications in Electronic Speckle Pattern Interferometry and Laser Doppler Vibrometry
}

Viswanath Bavigadda

Technological University Dublin

Emilia Mihaylova

Technological University Dublin, emilia.mihaylova@tudublin.ie

Raghavendra Jallapuram

Technological University Dublin, mrutic@gmail.com

See next page for additional authors

Follow this and additional works at: https://arrow.tudublin.ie/cieocon2

Part of the Optics Commons

\section{Recommended Citation}

Bavigadda, V., Mihaylova, E. \& Jallapuram, R. (2010). Design and Fabrication of Holographic Optical Elements for Applications in Electronic Speckle Pattern Interferometry and Laser Doppler Vibrometry. Proceedings Eighth International Conference on Vibration Measurements by Laser Techniques: Advances and Applications, vol. 7098. doi:10.1117/12.803006

This Conference Paper is brought to you for free and open access by the Centre for Industrial and Engineering Optics at ARROW@TU Dublin. It has been accepted for inclusion in Conference Papers by an authorized administrator of ARROW@TU Dublin. For more information, please contact arrow.admin@tudublin.ie, aisling.coyne@tudublin.ie,gerard.connolly@tudublin.ie. Funder: Enterprise Ireland

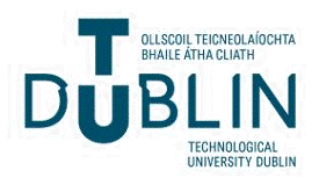


Authors

Viswanath Bavigadda, Emilia Mihaylova, Raghavendra Jallapuram, and Vincent Toal

This conference paper is available at ARROW@TU Dublin: https://arrow.tudublin.ie/cieocon2/5 
2010-01-01

\section{Design and Fabrication of Holographic Optical Elements for Applications in Electronic Speckle Pattern Interferometry and Laser Doppler Vibrometry}

Viswanath Bavigadda

Dublin Institute of Technology

Emilia Mihaylova

Dublin Institute of Technology, emilia.mihaylova@dit.ie

Raghavendra Jallapuram

Dublin Institute of Technology, Raghavendra.Jalla@finisar.com

Vincent Toal

Dublin Institute of Technology, vincent.toal@dit.ie

\section{Recommended Citation}

Bavigadda, V., Mihaylova, E., Jallapuram, R., Toal, V.: Design and Fabrication of Holographic Optical Elements for Applications in Electronic Speckle Pattern Interferometry and Laser Doppler Vibrometry. Proceedings Vol. 7098 Eighth International Conference on Vibration Measurements by Laser Techniques: Advances and Applications, Enrico Primo Tomasini, Editors, 70980O. DOI: 10.1117/ 12.803006 


\title{
Design and fabrication of holographic optical elements for applications in electronic speckle pattern interferometry and laser Doppler vibrometry \\ Viswanath Bavigadda*, Raghavendra Jallapuram, Emilia Mihaylova, Vincent Toal \\ Centre for Industrial and Engineering Optics, Dublin Institute of Technology, \\ Kevin Street, Dublin 8, Ireland. \\ *Corresponding author e-mail:viswanath.bavigadda@student.dit.ie
}

\begin{abstract}
An important area of application of holographic optical elements (HOEs) is in optical and electronic speckle pattern interferometry. The design, fabrication and characterization of holographic optical elements (HOEs) for electronic speckle pattern interferometry are presented. Reflection HOEs (RHOEs) were fabricated for use in electronic speckle pattern interferometers (ESPI) and laser Doppler vibrometers (LDV). The HOE-based interferometer is sensitive to outof-plane displacements only. The results obtained are promising for future applications of the system for modal analysis.
\end{abstract}

Key words: Holographic optical elements (HOEs), speckle interferometry, out of plane ESPI, Laser Doppler Vibrometry.

\section{INTRODUCTION}

ESPI is also known as TV holography or Digital holography. The use of speckle pattern interferometry to detect vibration modes was reported by Archbold et al (1969) [1]. TV detection and electronic filtering technique have been combined with the principle of speckle interferometry to give a flexible system [2,3, and 4]. ESPI has many advantages as it uses the wavelength of light as a measuring unit. The non-contact nature of the method is an added advantage when vulnerable surfaces are studied or when health and safety is an issue, as in food and pharmaceutical industries.

Holographic optical elements (HOEs) are lenses, mirrors, gratings, prisms and beam splitters made by holographic methods. They can be used as alternative optical elements to expensive conventional optical elements. ESPI is a full field measurement technique for studying the deformation pattern of an object surface. Random speckles are used as carriers from which phase information can be extracted. Our ESPI system consists of only HOEs. The basic idea in the HOE based ESPI system is the use of a speckle reference wave, which is stored in a holographic optical element [5]. Incorporating a reflection HOE in an ESPI system makes the interferometer very compact.

Commonly, holographic optical elements (HOE) are used at a wavelength differing from the recording wavelength, because the photographic emulsions used for recording the HOE, are not sensitive to light in the near infrared spectral range where most semiconductor lasers operate. Several authors have discussed ray tracing methods for analysing and designing HOEs [6]. In this paper we present some simple formulae for designing HOEs to be recorded at one wavelength for use at another. We record HOEs at $633 \mathrm{~nm}$ red laser and reconstruct with a near infra red $763 \mathrm{~nm}$ diode laser which can be wavelength modulated for phase shifting to facilitate detailed fringe analysis. In the theoretical analysis we neglect material shrinkage and aberrations.

In an out-of-plane sensitive ESPI system, the CCD camera should be positioned with its optical axis exactly perpendicular to the surface of the object under inspection which should be illuminated normally. 


\section{THEORY}

\subsection{Reflection holographic optical element}

The sign conventions followed in the theoretical derivation are shown in fig. 1. The normal to the surface from left to right i.e. $+\mathrm{Z}$ axis, is the reference direction $\left(0^{0}\right)$. Angles are measured with respect to this axis. In the case of a reflection hologram interfering beams approach the photosensitive layer in opposite directions, so the fringe angle is always around $90^{\circ}$ with respect to the $+Z$ axis.

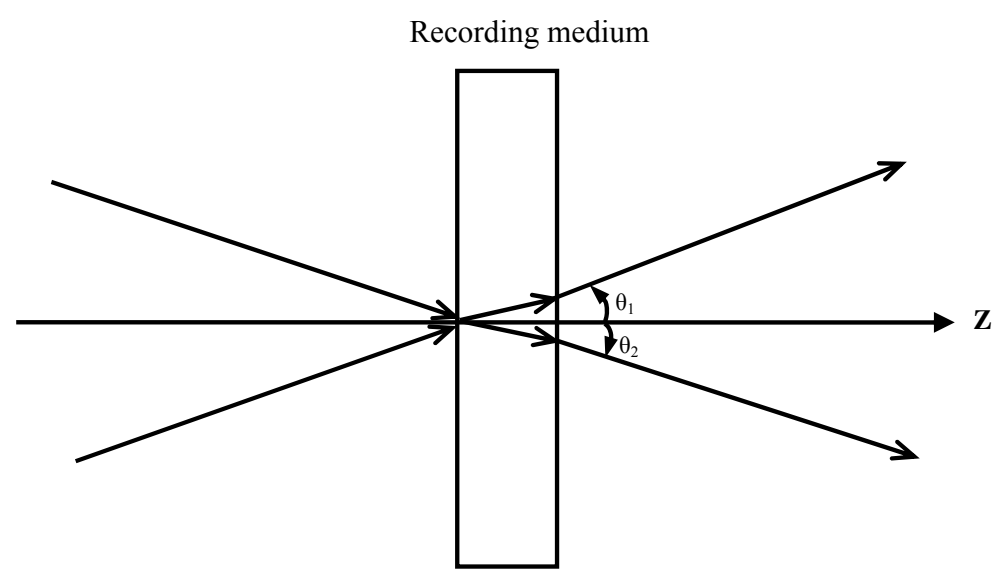

Fig. 1 Sign convention diagram for the measurement of angles of the rays

A specularly reflected ray from a diffusively reflecting flat object tilted at angle $\alpha$ to the $+z$ axis will renter the photosensitive layer making angle $\theta_{2}$.

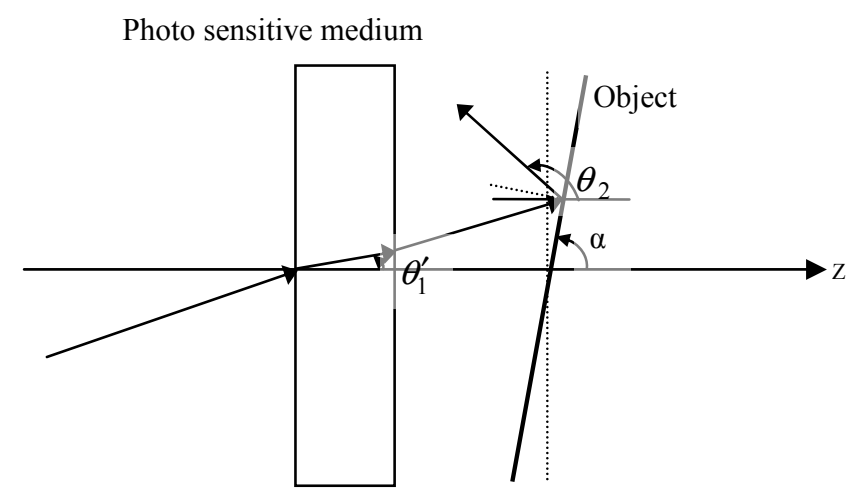

Fig. 2 Reflection hologram recording geometry in Denisyuk method

$\theta_{1}^{\prime}=$ the reference beam angle with respect to $+Z$ axis inside the medium

$\alpha=$ tilt of the object measured with respect to $+Z$ axis.

$\theta_{2}=$ The angle made by the object beam with respect to $+Z$ axis is obtained from the above geometry (fig.2) as

$\theta_{2}=180^{\circ}+\theta_{1}-2 \alpha$

$\theta_{1}^{\prime}, \theta_{2}^{\prime}$ are calculated using Snell's law and $\theta_{i}$ is the half of the inter-beam angle. 
Hence spacing d between the fringes $=\frac{\lambda}{2 \mu \cos \left(\frac{\theta_{1}^{\prime}+\theta_{2}^{\prime}}{2}\right)}$

$\mu=$ refractive index of the recording medium, for silver halide material $\mu=1.5$

$\lambda=$ recording laser wavelength

$\lambda^{\prime}=$ playback laser wavelength

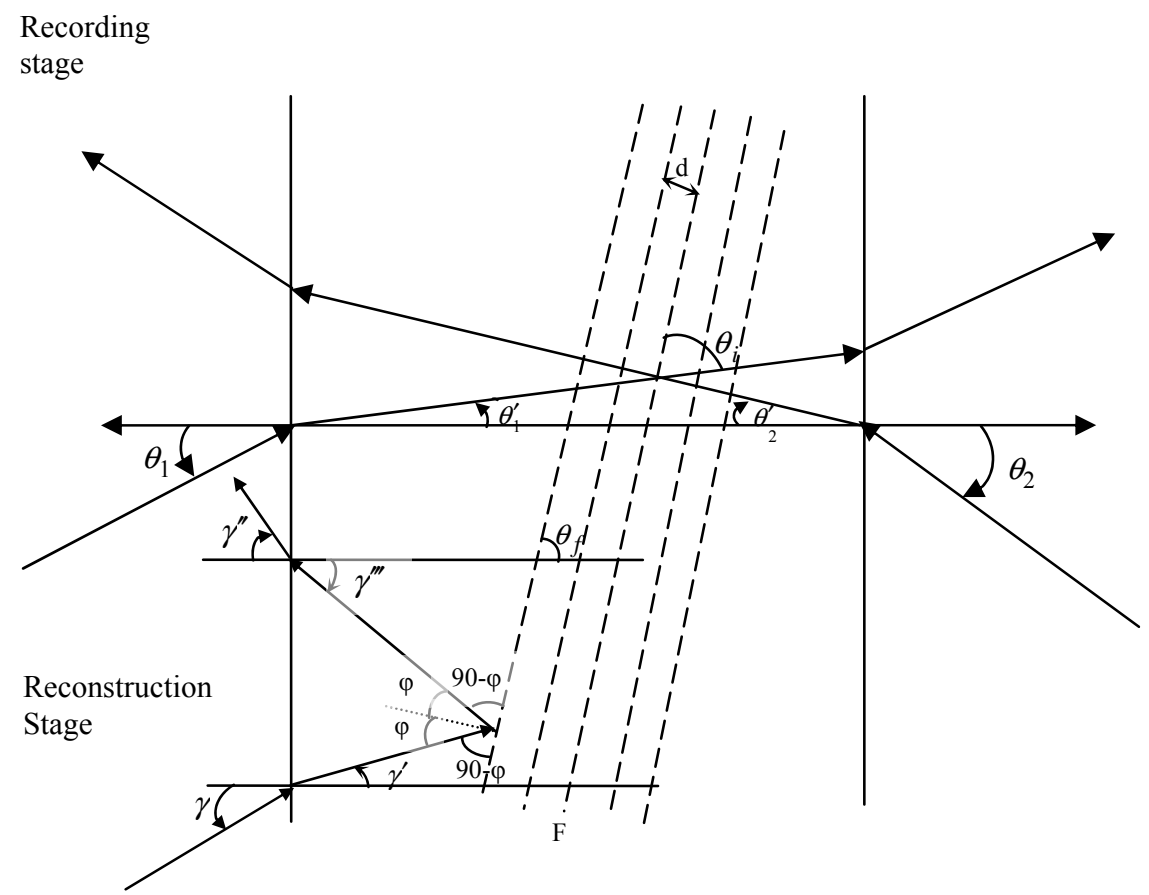

Fig 3 Recording and playback ray diagram inside the recording medium

The fringe angle $\theta_{f}=90+\left(\frac{\theta_{1}^{\prime}-\theta_{2}^{\prime}}{2}\right)$

For a successful reconstruction of the image we have to apply Bragg's law for $\lambda^{\prime}$

$2 d \cos \varphi=\frac{\lambda^{\prime}}{\mu}$

From equations (2) and (4)

Bragg angle $=\varphi=\cos ^{-1}\left[\frac{\lambda^{\prime}}{\lambda} \cos \left(\frac{\theta_{1}^{\prime}+\theta_{2}^{\prime}}{2}\right)\right]$

From fig. $3 \gamma^{\prime}=\varphi+\left(\frac{\theta_{1}^{\prime}-\theta_{2}^{\prime}}{2}\right)$

Hence applying Snell's law gives illumination angle 


$$
\begin{aligned}
& \gamma=\sin ^{-1}\left[\mu \sin \left\{\cos ^{-1}\left(\frac{\lambda^{\prime}}{\lambda} \cos \left[\frac{\theta_{1}^{\prime}+\theta_{2}^{\prime}}{2}\right]\right)+\frac{\theta_{1}^{\prime}-\theta_{2}^{\prime}}{2}\right\}\right] \\
& \gamma^{\prime \prime \prime}=\varphi-\left(\frac{\theta_{1}^{\prime}-\theta_{2}^{\prime}}{2}\right)
\end{aligned}
$$

Hence applying Snell's law gives diffracted beam direction.

$$
\gamma^{\prime \prime}=\sin ^{-1}\left[\mu \sin \left\{\cos ^{-1}\left(\frac{\lambda^{\prime}}{\lambda} \cos \left[\frac{\theta_{1}^{\prime}+\theta_{2}^{\prime}}{2}\right]\right)-\frac{\theta_{1}^{\prime}-\theta_{2}^{\prime}}{2}\right\}\right]
$$

Using equations (7) and (9) a MATLAB programme was written to calculate illumination and diffracted beam angles for a different wavelength from the wavelength used for recording. Also the recording angles $\theta_{1} \& \theta_{2}$ are chosen in such way that the reconstructed beam from the hologram travels almost along the $-\mathrm{Z}$ direction i.e. $\gamma^{\prime \prime} \approx 0^{\circ}$.

\section{EXPERIMENT}

\subsection{Recording reflection HOE’s}

The light from a He-Ne laser $(\lambda=633 \mathrm{~nm})$ was expanded by a spatial filter and collimated by a lens. The collimated beam was then partially transmitted by a glass coated silver halide emulsion layer (PFG-03M supplied by Geola). The light beam illuminating the surface of silver halide emulsion served as reference wave. The transmitted beam through the silver halide emulsion layer illuminated the object, a flat diffusely reflecting plate. The light scattered by the object illuminated the silver halide layer from the rear side serving as an object wave. These two beams interfered at the recording plane to produce reflection hologram.

\subsection{Material used for recording of HOE's}

The recorded silver halide layers were developed using the JD4 process [7].The plate was developed for 20secs in a solution containing equal parts of (JD4-A) and (JD4-B), then rinsed with water followed by bleaching and finally agitated in distilled water containing 2 drops of Kodak Photoflo per litre. Then the holograms were allowed to dry naturally.

\subsection{ESPI system}

The optical set-up for the ESPI system using a Reflection HOE is presented in Fig.4. The reflection holographic optical element (RHOE) of a flat diffusely reflecting surface generates the speckled reference beam. On reconstruction, a diffuse beam of laser light is produced to act as a reference beam in the ESPI system. Undiffracted light passing through the RHOE serves to illuminate the object beam. Silver halide layers were recorded in Denisyuk method at $633 \mathrm{~nm}$. The recording angles were $\theta_{1}=66^{\circ}, \theta_{2}=50^{\circ}$ which corresponds to the object tilt $\alpha=98^{\circ}$. In the reconstruction stage an IR diode laser $763 \mathrm{~nm}$ was used to illuminate the RHOE. The probing angle was $\gamma=10^{\circ}$ and diffracted beam angle $\gamma^{\prime \prime}$ relative to the $-Z$ axis was approximately $0^{\circ}$. Since the object is illuminated at $10^{\circ}$ to the normal there is some sensitivity to in-plane motion and the phase difference due to the displacement is given by [8]

$$
\begin{aligned}
& \delta=\frac{2 \pi}{\lambda^{\prime}}\left[v\left(\sin \gamma-\sin \gamma^{\prime \prime}\right)+w\left(\cos \gamma+\cos \gamma^{\prime \prime}\right)\right] \\
& \delta=\frac{2 \pi}{\lambda^{\prime}}[v(0.17)+w(1.98)]
\end{aligned}
$$

Where $\delta$ =phase difference before and after deformation, and $v, w$ are in and out of plane displacement components respectively. So our interferometer is sensitive primarily to out-of-plane displacements of the object. The relative intensities of the beams could be adjusted by slight rotation of the HOE. 


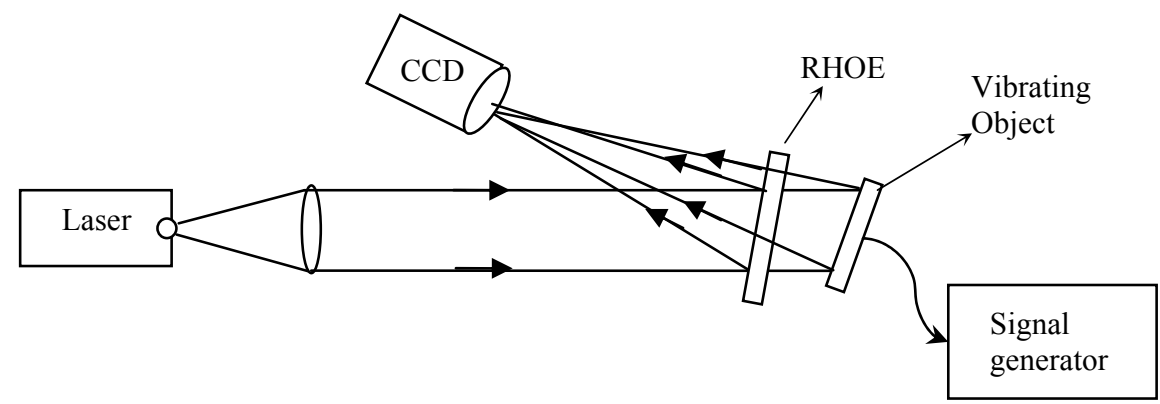

Fig. 4 Out-of-plane ESPI system using RHOE

\section{RESULTS}

A vibrating circular aluminium diaphragm of diameter $4 \mathrm{~cm}$ used as an object and it gives different resonant modes at different exciting frequencies. Some of the modes are shown in fig.5; these were obtained by deliberately altering the drive voltage after capturing a time averaged frame, thus subtracting interfograms at different voltages from one another.

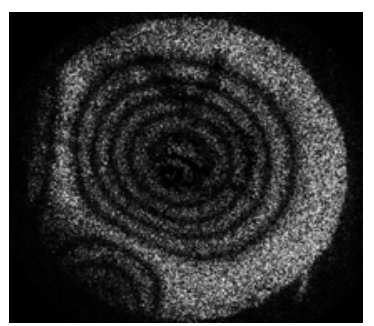

$4.2 \mathrm{kHz}$

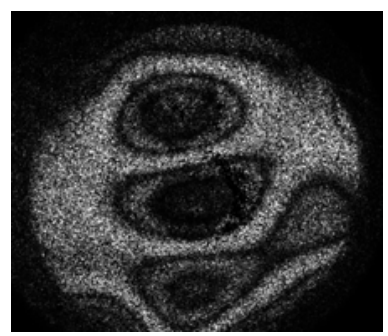

$8.2 \mathrm{kHz}$

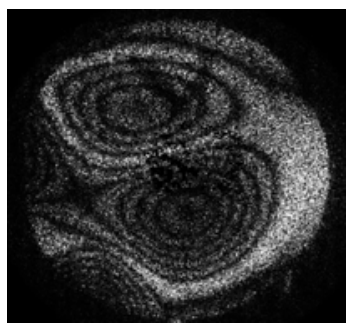

$5.1 \mathrm{kHz}$

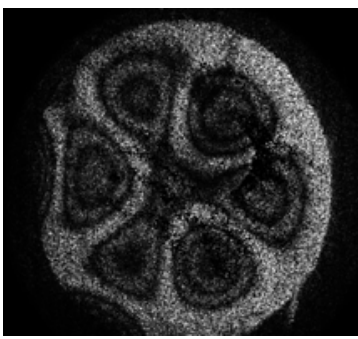

$10 \mathrm{kHz}$

Fig.5 ESPI vibration modes of an aluminum diaphragm. The amplitude of the sinusoidal signal driving the loudspeaker is $6 \mathrm{~V}$

\section{CONCLUSIONS}

We have successfully recorded reflection HOEs in visible laser light for reconstruction in the near IR. The experimental and theoretical values of the optimal angles of illumination and diffraction from the HOE at the near IR wavelength are in approximate agreement with each other. This enables us to construct a simple out-of-plane sensitive ESPI system which we have used to obtain vibrational mode patterns of a loudspeaker using subtraction method. The use in the ESPI system of a near IR, distributed feedback (DFB) laser diode whose wavelength can be modulated will allow phase 
shifting for fringe analysis purposes. The use of such a laser will also enable us to build and out-of-plane sensitive Laser Doppler Vibrometer incorporating a reflection HOE.

\section{ACKNOWLEDGEMENTS}

Acknowledgements are made to Technology Development Programme of Enterprise Ireland supported by the Irish Government.

I would like to acknowledge Technical sector Strand I for funding and technical support from IEO. I would like to sincerely thank my colleague Nitesh Pandey for his help in writing MATLAB code.

\section{REFERENCES}

[1] Butters J.N.and Leendertz, J.A., J. Phys. E, Scientific Instruments: 4 272-9 (1971)

[2] Macovski A, Ramsey S D and Schaefer L F, b Meas. Control 4 349-54 (1971).

[3] Guntaka S R, Toal V and Martin S, Appl.Opt. 41, 7475-9(2002)

[4] Ole J. Lokberg, "ESPI--The ultimate holographic tool for vibration analysis?" J. Acoust. Soc.Am.75 (6), 1783-91 (1984)

[5] Sridhar Reddy Guntaka, Venteseslav Sainov, Vincent Toal, Suzanne Martin, Tsveta Petrova and Jana Harizanova, "Compact electronic speckle pattern interferometer using a near infrared diode laser and a reflection holographic optical element”, J.Opt.A:Pure.Appl.Opt.8 182-188 (2006)

[6] Champagne, E.B., "Non-paraxial imaging magnification, and aberration properties in holography", J.Opt.Soc. Am. A, 57, 51-55. (1967)

[7] http://www.integraf.com/Downloads/JD-4\%20Instructions\%20_v04.11.pdf

[8] Rastogi.P.K (Editor), [Optical measurement techniques and applications], Artech House Inc. Boston \& London, p159 (1997). 